\title{
Modeling the benefits of pamidronate in children with osteogenesis imperfecta
}

\author{
Robert Lindsay \\ Department of Medicine, Columbia University, Helen Hayes Hospital, West Haverstraw, New York, USA \\ J. Clin. Invest. 110:1239-1241 (2002). doi:10.1172/JCI200217051.
}

\begin{abstract}
Osteogenesis imperfecta (OI) represents a phenotypically heterogeneous group of conditions that result from a number of genetic defects in the synthesis of type 1 collagen (1). The clinical expression is primarily that of bone fragility, but other tissues can also be involved, including teeth, ligaments, and joints. The clinical classification system devised by Sillence and colleagues (1) describes most cases of OI, but recent modifications to this system encompass additional forms of the disease. Phenotypes range from a mild form that may cause only a few childhood fractures with no deformity (type I) to the most severe form (type II), which is usually lethal in the perinatal period due to multiple fractures and marked bony deformities. Types III and IV are both associated with deformity and are the most difficult to manage clinically. Each phenotype results from one or more defects in collagen production, almost all inherited in an autosomal dominant fashion. At least 200 disease alleles have been reported in the COL1A1 or COL1A2 genes, and qualitative collagen defects are found to alter the structure of type 1 collagen in all forms of the disease (2). However, the milder and most commonly seen clini-

$\alpha-1$ RNA. Recently, two variants of OI (labeled types V and VI), which involve mutations affecting genes other than those encoding type 1 collagen chains, have been described $(3,4)$.

The genetics of OI and the biochemical abnormalities of type 1 collagen associated with increased fracture risk have become increasingly well understood. However, the metabolic consequence of OI on the skeleton, as well as its contribution to fracture risk, still remains poorly characterized, in part because of the difficulty in evaluating skeletal homeostasis in children. For this reason, it was difficult to anticipate the effects of treating OI patients with pamidronate, an agent that has been employed as a means to reduce fractures and deformity $(5,6)$. Pamidronate would be expected to reduce bone remodeling but have no effect on collagen expression in OI patients or many of the immediate sequellae of incorporating mutant collagen chains into the bone matrix. Nevertheless, the elegant histomorphometric evaluations of iliac crest biopsies by Rauch et al. (7) in this issue of the JCI confirm the utility of this approach and begin to shed some light on the mechanisms by which pamidronate acts.
\end{abstract} cal variants of $\mathrm{OI}$ result from premature stop codons, or out-of-frame RNA splicing defects that lead to a reduction in the absolute level of type 1 collagen

\footnotetext{
Address correspondence to: Robert S.

Lindsay, Department of Medicine, Columbia University, Helen Hayes Hospital, Route 9W, West Haverstraw, New York 10993, USA. Phone: (845) 786-4494; Fax: (845) 786-4878;

E-mail: lindsayr@helenhayeshosp.org.

Conflict of interest: No conflict of interest has been declared.

Nonstandard abbreviations used:

osteogenesis imperfecta (OI).
}

\section{Bone growth and metabolism in OI} Until recently, the management of OI was limited to orthopedic intervention and aggressive rehabilitation in attempts to maximize patient skills for independent living, especially among those with deforming disease (types III and IV, and severe type I cases) (2). Arguably, this heterogeneous group of disorders might be amenable to gene therapy $(2,8)$, but hope for this approach rests with the possibility of modifying the qualitative abnormali- ties in collagen synthesis by suppressing expression of the mutant RNAs. Neither antisense oligonucleotide nor ribozyme approaches have reached clinical practicality, and management remains a clinical problem, often requiring intensive team approaches to the long-term management of affected individuals.

The benefits of the amino-bisphosphonate pamidronate on bone density and fracture rate in children with OI appeared to offer hope, but the efficacy of this agent has seemed counterintuitive, given its known primary action on bone remodeling. Still, OI is more than simply an insufficient amount of collagen or defective collagen structure. Aberrant post-transcriptional modifications and incorporation of the mutant chain into a collagen triple helix affect the intrinsic stability of the trimer. Subsequent metabolism of the weakened bone matrix likewise contributes to skeletal fragility $(2,8)$. Even beyond the structural abnormalities that result from incorporating abnormal collagen fibrils into triple helices or from the synthesis of helices lacking the normal stoichiometry of COL1A1 and COL1A2 collagen chains, these mutations can alter cellular function directly or indirectly. Cortical modeling of bone, a requirement for overall skeletal growth (9), may be impaired, leaving the skeletons of OI patients smaller than those of aged-matched individuals, even after correcting for stature (2). Since size is an important determinant of bone strength, the result is an increased propensity for fracture. There is also impairment of bone formation, especially obvious in the formation of primary trabeculae, a process that occurs by endochondral ossification or cancellization of cortical bone. 


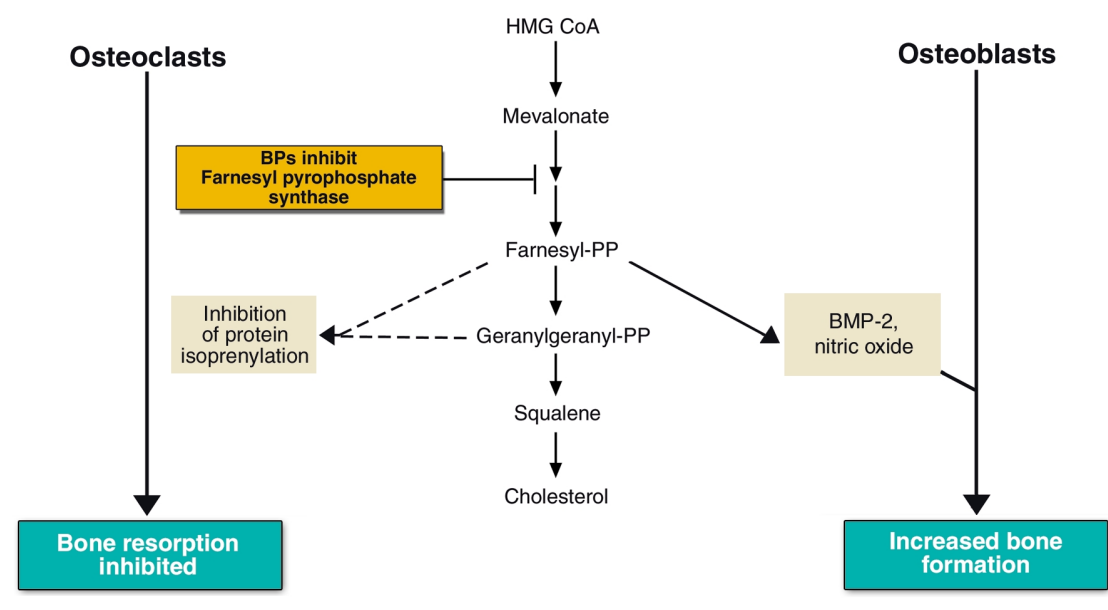

Figure 1

Proposed mechanism of bisphosphonate (BP) action. BPs inhibit a key step in the hydroxymethylglutaryl conenzyme A (HMG-CoA) reductase pathway through the inhibition of farnesylpyrophosphate synthase. Inhibition of protein isoprenylation results in osteoclast apoptosis and inhibition of osteoclast-mediated bone resorption. GTP, guanosine $5^{\prime}$-triphosphate; PP, pyrophosphate; BMP-2, bone morphogenetic protein-2.

As a result of defective collagen biosynthesis, the osteoblasts of children with OI produce less bone than is required. The balance between bone resorption and formation, which normally favors formation and allows for thickening and strengthening of trabeculae during childhood (9), is tipped toward resorption. The resulting thinner primary trabeculae are then preferentially removed, in part because of the instability of the collagen, but also perhaps because of greater than normal physical strain within the bone, which may induce the resident osteocytes to activate local bone remodeling. Thinner trabeculae are also likely to suffer microdamage because of higher strain associated with the stress of normal activities (on less bone tissue), especially where fewer trabeculae survive. This microdamage attracts targeting remodeling (10). In addition, the net effect of increased remodeling is in itself an increase in damage, as remodeling sites become stress risers - points of focus for strain at which microfractures become more likely (10). All of these mechanisms may contribute to the elimination of primary trabecular structures and thus to weaker bone. Therefore, growing children with OI have small, thin bones with few trabeculae, thin cortices, and high remodeling rates. The consequences are deformities and a greatly increased risk of fractures. tein tethering to the cell membrane and

\section{Figure 2}

inhibits biological function, ultimately resulting in osteoclast apoptosis (Figure 2). In addition to their effect on osteoclast survival, bisphosphonates may also interfere with osteoclastmediated bone resorption, since this process can be blocked in vitro at bisphosphonate doses lower than those required to induce apoptosis (12).

Due to the possibility of long-term effects of bisphosphonate treatment in growing children, Rauch et al. (7) treated only the most severely affected individuals: those with more than three fractures per year for the previous two years. Consequently, most of the subjects in this study had OI types III and IV or were among the more severely affected type I patients. They found that pamidronate therapy increased cortical width but not overall iliac crest width. The resulting improvement in cancellous bone volume was apparently entirely accounted for by increased trabecular number. As expected, pamidronate reduced bone remodeling in cancellous bone and on the endocortical surface of the external cortex but had little effect on the periosteal or endocortical surface of the inner cortex. To account for increased cortical width, Rauch and colleagues propose that modeling of cortical bone is insignificantly affected by pamidronate. However, since bone size is not altered and no change occurs in the rate of cortical bone formation on the endocortical surface, it appears more likely that the drug acts at the endocortex to favor bone formation over resorption during remodeling. Pamidronate also reduces activation of new remodeling cycles

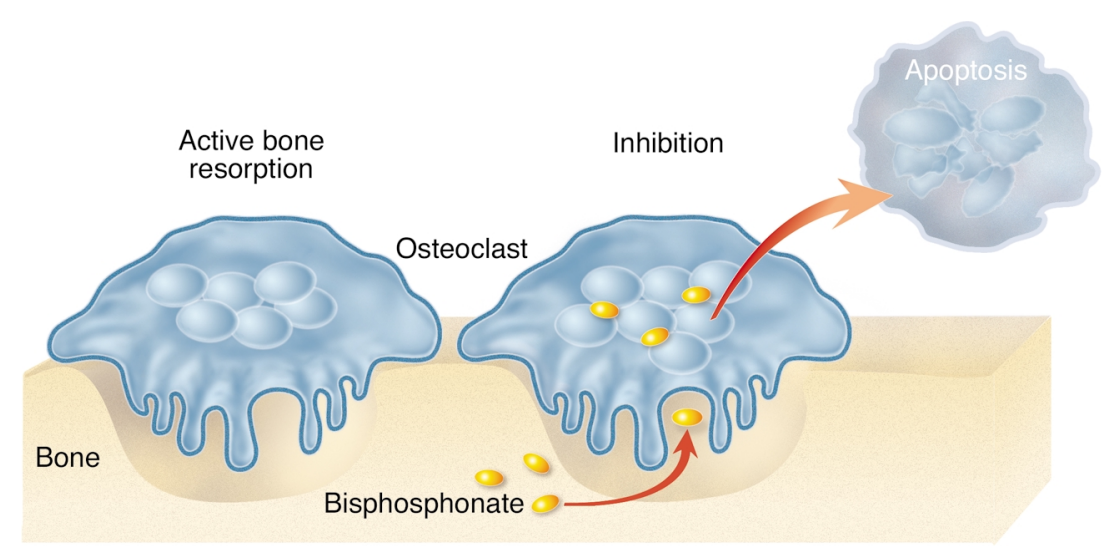

by Ken Beauchamp J. Clin. Invest.

Active osteoclasts mediate bone resorption. Osteoclast absorption of bisphosphonates results in the inhibition of osteoclast function and osteoclast apoptosis. 
by reducing the population of cells committed to becoming mature osteoclasts. The observed increase in trabecular number is also a likely result of less frequent bone remodeling. Because pamidronate allows for an increase in trabecular number, individual trabeculae are presumably under less strain and show less microdamage. As a result, targeted resorption of primary and secondary trabeculae and targeted bone remodeling both decline. This reduction in remodeling within primary trabeculae most likely accounts for the higher amount of calcified cartilage and reduced marrow fibrosis seen during treatment.

\section{Risks and benefits}

The clinical effects of pamidronate are marked $(5,6)$. In children, fracture rates can be reduced by $60 \%$, especially in those under three years of age who mostly present with type III. In older, prepubertal girls an $80 \%$ reduction in fracture risk is seen. Among children who fracture two to four times per year, this benefit is dramatic. Furthermore, it appears that fracture healing and growth are not affected.

Nevertheless, since bisphosphonates accumulate in the bone and residual levels are measurable after many years, the long-term safety of this approach is unknown and remains a matter of concern. Given a high enough skeletal load, bisphosphonates might continue to exert effects as they are gradually released from bone even after treatment is discontinued. Furthermore, while remodeling clearly continues in pamidronate-treated children, it is markedly reduced. It remains unclear whether the consequential increase of calcified cartilage has a detrimental effect. However, as Rauch and colleagues indicate, until long-term safety data are available, pamidronate intervention should be reserved for those for whom the benefits will clearly outweigh the risks. The availability of oral bisphosphonates that can be given once per week (13) may allow easier administration of maintenance therapy. Bisphosphonates might now also be considered as a possible intervention for postmenopausal women with mild OI, who are at an increased risk of fractures since osteoporosis becomes superimposed on their primary disease.

Bisphosphonate therapy clearly represents a major advance in the treatment of children with severe OI. The clinical course and attendant morbidity for many children with severe OI is clearly improved with its judicious use. Although several approaches aiming to correct the genetic abnormalities underlying OI are being pursued, their clinical practicality still lies in the future. Bisphosphonate treatment now most likely sets the standard against which other such novel therapies will be assessed.

1. Sillence, D.O., Senn, A., and Danks, D.M. 1979 Genetic heterogeneity in osteogenesis imperfec- ta. J. Med. Genet. 16:101-116.

2. Rowe, D.W. 2002. Osteogenesis imperfecta. In Principles of bone biology. 2nd edition. J.P. Bilezikian, L.G. Raisz, and G.A. Rodan, editors. Academic Press. San Diego, California, USA. 1177-1193.

3. Glorieux, F.H., et al. 2000. Type V osteogenesis imperfecta: a new form of brittle bone disease. J. Bone. Miner. Res. 15:1650-1658.

4. Glorieux, F.H., et al. 2002. Osteogenesis imperfecta type VI: a form of brittle bone disease with a mineralization defect. J. Bone Miner. Res. 17:30-38.

5. Glorieux, F.H., et al. 1998. Cyclic administration of pamidronate in children with severe osteogenesis imperfecta. N. Engl. J. Med. 339:947-952.

6. Astrom, A., and Soderhall, S. 2002. Beneficial effect of long term intravenous bisphosphonate treatment of osteogenesis imperfecta. Arch. Dis. Child. 86:356-364.

7. Rauch, F., Travers, R., Plotkin, H., and Glorieux, F.H. 2002. The effects of intravenous pamidronate on the bone tissue of children and adolescents with osteogenesis imperfecta. J. Clin. Invest. 110:1293-1299. doi:10.1172/JCI200215952.

8. Dawson, P.A., and Marcus, J.C. 2000. Osteogenesis imperfecta. In The genetics of osteoporosis and metabolic bone disease. M.J. Econs, editor. Humana Press. Totowa, New Jersey, USA. 75-93.

9. Duan, Y., Turner, C.H., Kim, B.T., and Seeman, I. 2001. Sexual dimorphism in vertebral fragility is more the result of gender differences in age related bone gain than bone loss. J. Bone Miner. Res. 16:2267-2275

10. Dempster, D.W. 1999. New concepts in bone remodeling. In Dynamics of bone and cartilage metabolism: principles and clinical applications. M.J. Seibel, S.P. Robins, and J.P. Bilezikian, editors. Academic Press. San Diego, California, USA. 261-273.

11. Dunford, J.E., et al. 2001. Structure-activity relationships for inhibition of farnesyl diphosphate synthase in vitro and inhibition of bone resorption in vivo by nitrogen-containing bisphosphonates. J. Pharmacol. Exp. Ther. 296:235-242.

12. Hawasy-Nagy, J.M., Rodan, G.A., and Reszka, A.A. 2001. Inhibition of bone resorption by alendronate and Risedronate does not require osteoclast apoptosis. Bone. 29:553-559.

13. Brown, J., et al. 2002. The efficacy and tolerability of risedronate once-a-week for the treatment of osteoporosis. Calcif. Tissue Int. 71:103-111. 